\title{
APROXIMACIÓN A LA ACTITUD DE DOCENTES Y ALUMNOS SOBRE ANDALUCÍA
}

Fernando RUIZ MORALES*

Sin duda, el campo educativo es uno de los principales focos proveedores de modelos de identificación para los sujetos y grupos. La escuela es, así, uno de los agentes decisivos para la formación de la conciencia étnica y nacional. En Andalucía, después de un período suficientemente dilatado de autonomía, es preciso revisar el papel que el sistema educativo está jugando en este sentido. Adentrándonos en esto, hemos revisado algunos elementos significativos (tarea en la que seguimos), como son la legislación educativa y los libros de texto ${ }^{2}$. Hemos realizado también una aproximación mediante un cuestionario para docentes de Primaria y Secundaria, de varios colegios e institutos de Sevilla (capital y provincia), y revisado algunos escritos de alumnos sobre Andalucía, todo ello en 1998. Estas facetas son las que expondremos aquí.

\section{LOS DOCENTES}

Aunque con todas las precauciones del mundo, que son siempre pocas cuando de cuestionarios se trata, vamos a exponer primero algunas respuestas significativas de los docentes ${ }^{3}$.

* Licenciado en Geografía e Historia, Secciónd e Antropología Social, por la Universidad de Sevilla.

1. Grupo de Estudio sobre las Identidades Socioculturales en Andalucía (Universidad de Sevilla).

2. Trabajo aún no publicado, salvo en aspectos parciales: Ruiz Morales (1999a y 1999b).

3. Al cuestionario han respondido 126 docentes de 9 centros de Primaria (en 6 de los cuales se imparte también el primer Ciclo de Secundaria). De estos centros de Primaria, 3 son de Sevilla, 2 de Dos Hermanas, y uno de Los Rosales, Las Cabezas de San Juan, El Viar y Gerena; en cuanto a los institutos, ha sido 5; 4 de ellos son de Sevilla y uno de San Juan de Aznalfarache. Este cuestionario lo hemos planteado de forma un tanto atípica, con un «espíritu» poco sociológico: junto a preguntas más o menos objetivas y cerradas, hay otras muy abiertas y enunciados lo suficientemente ambiguos como para intentar apreciar no tanto un hecho o una opinión como una reacción del encuestado. Esto (que puede ser criticable, y lo asumimos) resta valor estadístico al cuestionario, pero 
En primer lugar, los docentes parecen más vinculados a la Administración lo cal, es decir, al Ayuntamiento y sus servicios, que a la Junta, puesto que acuden : aquél más a la hora de realizar actividades (el 34'9\% y el 21'4\% respectivamen te); ahora bien, recurren, más que al Ayuntamiento, a otras entidades (el 42’1\%) empezando por el propio centro de trabajo.

Los docentes consideran que Andalucía tiene, o bien el peso justo en el currí culo (47'6\%), o bien que tiene poco peso en el mismo (42'9\%). Es insignificant la cantidad de los que piensan que demasiado o ninguno. El 72 ' $2 \%$ de 10 : encuestados no ha realizado cursos de formación sobre cultura andaluza en lo: últimos tres años. El 20'9\% de ellos entiende que porque la oferta es escasa, y has un significativo 34'1 \% de aquéllos que no los ha hecho por tener otras prefe. rencias.

En cuanto a los libros de texto, es preciso apuntar algo a partir de las respues. tas de los docentes. Para el 42'9\% los libros de texto dedican suficiente espacio : Andalucía, mientras que el $40{ }^{\prime} 5 \%$ entiende que éste es insuficiente. En líneas generales, la tendencia en la respuesta a esta cuestión se corresponde con la mencionada valoración sobre el peso que Andalucía tiene en el currículo, aunque ur notable 15 ' $1 \%$ no responde aquí. En cuanto a la valoración de la imagen que los libros ofrecen de Andalucía, el 31'7\% del total la considera buena, correcta o suficiente, mientras que el $28^{\prime} 6 \%$ entiende que es pobre en contenidos y el 7 ' $9 \%$ inadecuada. El 4'8\% la considera mejorable o variable según los casos, y el 2’4\% la ve demasiado chauvinista o excesivamente nacionalista. Si excluimos estos dos últimos grupos, volvemos a observar un equilibrio entre dos tendencias dominantes polarizadas, pero ninguna extrema: los que lo valoran en general bien (el mencionado $31^{\prime} 7 \%$ ) y los que se muestran disconformes o críticos (el 36'5\%). Por otra parte, en los dos primeros Ciclos de Primaria, el libro de texto (de Conocimientc del Medio o de Lengua) juega un papel imprescindible para el $36{ }^{\prime} 1 \%$, y no es central, sino complementario o auxiliar, para el 61'1\% (el 2'8\% restante no responde). Todos lo utilizan. En el tercer Ciclo de Primaria (cursos $5^{\circ}$ y $6^{\circ}$ ) es imprescindible para muchos más: el 63’7\%. Son, pues, mucho más relevantes, lo que contribuye a justificar una atención especial a este tema. Para el 27’3\% de los docentes de este Ciclo son auxiliares, y todos los utilizan. En Secundaria (en las materias de Ciencias Sociales y Lengua) son auxiliares para el $21^{\prime} 7 \%$ y no los utilizan el 4’3\% Para el 52’2\% son imprescindibles, y no sabemos la opinión del resto. Según manifiestan, en la mayoría de los libros $\left(70^{\prime} 6 \%\right)^{4}$ sale la bandera de

abre sugerencias a las que no renunciamos. Por otra parte, la precaución que hay que tener en todos los cuestionarios se acentúa aquí no sólo por esto, sino también porque la selección de la muestra ha sido aleatoria, por lo que perdemos muchas perspectivas, empezando por las de aquellos que rechazan abiertamente tratar los temas planteados en el mismo y terminando por la representatividad estadística. Sin embargo, vamos a apuntar, a pesar de esto, datos, porque quizás en algunos casos pueden resultar, si no representativos, sí sugerentes hasta el punto de poder inferirse tendencias predominantes que son susceptibles de servir de punto de arranque para plantear algunas hipótesis a comprobar de manera sistemática. 
Andalucía, pero en el resto $\left(29^{\prime} 4 \%\right)$ no, lo que es muy notable. El himno sólo aparece en el $29^{\prime} 4 \%$ de los libros (especialmente en Primaria), y dedican a Andalucía, según los docentes, al menos la tercera parte de sus páginas menos de la mitad de ellos (41'2\%). Andalucía se presenta indistintamente con información dentro del tema principal (lo más corriente), con temas propios o en apéndices al final o tras el tema principal. Ningún libro (siempre de Conocimiento del Medio, Lengua y Literatura o Ciencias Sociales), según los docentes, ofrece una imagen deformada de Andalucía. El 70'6\% de los libros refuerzan la idea de que Andalucía tiene identidad propia, otro tanto de los libros combate el racismo, y el 64'7\% informa bien sobre el regionalismo andaluz y la autonomía. Son interesantes tanto estas relativamente altas cifras como el dato anterior, puesto que parecen demostrar un no muy alto nivel de exigencia, a la hora de enjuiciar los libros, por parte de muchos docentes. No obstante, no hay unanimidad en los criterios, puesto que hemos observado respuestas muy distintas para libros del mismo Ciclo y editorial. Depende esto, en gran medida, de los planteamientos del docente sobre este tipo de temas.

Son pocos libros los que, al parecer de los docentes, fomentan la identidad europea por encima de cualquier otra (el 11'8\%), lo que en buena medida demuestra una creciente asunción de ésta por parte de los mismos. El 41'2\% consideran que fomentan preferentemente la española, sobre todo en Lengua.

Más reveladoras resultan otras informaciones. El 66’7\% organizó algo en clase con motivo del 28 de Febrero de 1998, lo que, superando ampliamente la mitad, nos deja más de un 30\% para los que tal efemérides no mereció mayor relieve en clase. A nivel de Centro la cosa cambia, puesto que sólo uno (un Instituto) no organizó nada. Un acto con la bandera ${ }^{5}$, el himno, recitales, exposiciones de trabajos y desayunos de pan con aceite fueron las actividades más frecuentes.

La presencia de Andalucía en clase con temas, actividades o sesiones específicas se da en el 38'4\% de los casos, mientras que para el 28'6\% Andalucía está en sus clases en tanto forma parte de otros temas o actividades. El $24{ }^{\prime} 6 \%$ considera que Andalucía está poco presente o sólo «regular» en sus clases, y el 7'1\% entiende que nada presente. Si sumamos estos últimos obtenemos un 31 '7\%, coincidente en general con la proporción de los que no hacen nada especial con motivo del Día de Andalucía.

La materia que imparten no debe enfocarse igual en Andalucía que en Madrid para el $54 \%$, porque (para el $63^{\prime} 6 \%$ de éstos) las peculiaridades y la identidad son distintas. Tras estos motivos, de tipo cultural, destacan (el 21'8\% de los que responden así) otros que podríamos considerar más técnicos: la necesidad de partir

4. Se ha considerado por editorial y ciclo (no curso), de la siguiente manera: de $1^{\circ}$ a $4^{\circ}$ de Primaria, tercer Ciclo de Primaria y ESO. Con una sola respuesta señalada por libro y ciclo, hemos considerado esa respuesta afirmativa para esa editorial, independientemente de que en otros casos la respuesta haya sido negativa.

5. Asistimos a uno, en el que junto a la bandera de Andalucía se lucía la de España. 
del entorno del alumno. Por contra, el 38 ' $1 \%$ piensa que debe enfocarse igual, es primer lugar porque la materia o los conceptos son iguales o universales (el 53' $1 \%$ de los que opinan esto), y en segundo lugar (el 18'75\%) para no discriminar, pro piciar encuentros o evitar "el peligro de los localismos».

La mayoría declara no tener en clase ningún símbolo identificativo de Andalu cía, España, Europa o la localidad (48'4\%). De Andalucía lo tiene el 30’9\%, dı España el $23{ }^{\prime} 8 \%$, de la localidad el $19^{\prime} 8 \%$ y de Europa el 12'7\%. Es normal qu haya en la misma clase símbolos de más de una de estas entidades. Para el 38'9\% no es necesaria la presencia de ninguno de estos símbolos. Más de la mitad, et cambio (el 52'4\%), ve necesarios símbolos de Andalucía (lo que contrasta con e $30^{\prime} 9 \%$ que en efecto los tiene), el $40^{\prime} 5 \%$ de la localidad (lo que nos ofrece tarn bién otro notable contraste), el 36'5\% de España y el 31\% de Europa (otro con traste considerable). A menudo, entienden que sería necesario más de un símbolo

Para el 41'2\% es deseable que la escuela fomente en los alumnos algún senti do de conciencia nacionalista andaluza, mientras que no lo es para casi otro tantc $\left(39^{\prime} 7 \%\right)$, y resulta indiferente para el $15^{\prime} 1 \%$. Ese $41^{\prime} 2 \%$ puede incluir una enor me variedad, que hemos comprobado relacionando esta respuesta con la dada : otras preguntas. Por tanto, preferimos más bien contemplar el grado de rechazc hacia el tema, que habría que cifrar en el referido $39{ }^{\prime} 7 \%$.

El acontecimiento histórico más positivo para Andalucía es, según opina e $31^{\prime} 7 \%$, la autonomía, el Estatuto o el referéndum del 28-F. Le siguen la époc: musulmana, pero muy de lejos $\left(7^{\prime} 9 \%\right)$, el descubrimiento de América y el comer cio con la misma ( $\left.7^{\prime} 1 \%\right)$, y la llegada de la democracia, la Expo 92 y el "hecho, de ser "crisol», cruce, mezcla o paso de culturas; cada uno de estos aspectos cons. tituye lo más positivo para el $4 \%$. Del total de acontecimientos o períodos señala. dos, el 30'7\% son acontecimientos que involucran básicamente a España, en lo: que Andalucía fue más bien una pieza del engranaje (descubrimiento de América llegada de la democracia, Expo, AVE, etc.). En cuanto al acontecimiento más negativo, se encuentra muy repartido. Los más citados son la Reconquista o episo. dios relacionados con sus consecuencias (el 13'5\% de los docentes) y la guerr civil o la dictadura franquista (11'1\%).

Los datos expuestos son los más generales y los más, digamos, imprescindi. bles. Podemos de ellos sacar algunas conclusiones provisionales sobre los docentes. Ante todo, hay dos posicionamientos mayoritarios aunque nada impermeables entre sí. Ninguno baja del 30\% de encuestados en cada aspecto. El primero seríc una postura de orientación hacia, al menos, la empatía respecto a Andalucía; organizar algo con motivo del 28-F, entender que no puede enseñarse igual aquí quє en Madrid ${ }^{6}$ y ver necesarios símbolos de Andalucía en clase son opciones de más

6. Aunque cuidado con esto: en un caso, entiende que el enfoque no puede ser igual pnrque aquí hay má: vicios y errores que corregir en el lenguaje empleado por los alumnos, y no pocos no han expuesto los motivos di su opción. 
de la mitad de los docentes. Considerar insuficiente el peso de Andalucía en los libros de texto y en los currículos, así como considerar necesario transmitir un sentimiento de conciencia nacionalista andaluza, lo hacen más del $40 \%$. Por su parte, más del $30 \%$ (pero menos del 40 ) son críticos con los libros de texto, dedican a Andalucía en sus clases sesiones o actividades específicas, tienen símbolos andaluces en clase y consideran el proceso autonómico como el hecho más positivo de nuestra historia. Por el lado opuesto, más de la mitad considera que no hay que transmitir sentimientos etnonacionalistas o son indiferentes a esta proposición, mientras que más del $40 \%$ ven que el peso de Andalucía en los currículos y en los libros de texto es suficiente; y más del $30 \%$ no consideran necesarios símbolos de Andalucía en clase, entienden que el enfoque de sus clases ha de ser igual aquí que en Madrid, ven buena la imagen dada por los libros de texto, Andalucía está poco presente en sus clases, destacan como hechos históricos más positivos acontecimientos que nos remiten básicamente a la historia de España, y no han celebrado nada en clase con motivo del Día de Andalucía.

Esta polarización, no extrema, no es tampoco rígida, puesto que en multitud de casos el mismo docente se alinea, según las cuestiones planteadas, en una o en otra. No es, por tanto, una polarización fuerte ni militante. La principal conclusión de esto es que hay una línea tenue y variable, graduada, y oscilante, en la práctica escolar, que va desde posturas de franco interés hacia Andalucía y conciencia al respecto, hasta la no consideración, e incluso abierta hostilidad, hacia el tema.

\section{LAS VARIABLES LUGAR DE NACIMIENTO Y NIVEL IMPARTIDO}

Hay dos variables, sin embargo, que nos ofrecen pistas interesantes. Lo que sigue es una descripción de esta muy somera encuesta a una mínima muestra, pero el peso de esas variables nos ha sorprendido. Nos referimos al lugar de nacimiento $\mathrm{y}$ al ciclo educativo impartido.

El peso de Andalucía en el currículo es el justo para el $44 \%$ de los docentes andaluces encuestados, y nada menos que para el 70'6\% de los no nacidos en Andalucía. Las diferencias, por tanto, son notables en relación con esta variable. En cuanto al nivel impartido (lo hemos dividido en dos: Primaria y primer Ciclo de Secundaria, por un lado -diremos, para simplificar, Primaria-; y Secundaria completa o segundo Ciclo de la misma, por otro $)^{7}$, no hay diferencias notables en esto.

7. Esta división se jnstifica porque quienes sólo imparten el Primer Cíclo de Secundaria suelen ser maestros de Primaria (independientemente de si sonlicenciados o no), y los que imparten el Segundo Ciclo de Secundaria 
En lo que respecta a la institución a la que acuden para organizar actividades, los de Primaria son los principales «clientes» de los Ayuntamientos (el 38'1\%), en mayor medida que los de Secundaria (24'1\%), que además recurren a éste tanto como a la Junta. No hemos observado relevancia del lugar de nacimiento en este tema.

En la consideración sobre el espacio dedicado a Andalucía en los libros de texto, volvemos a encontrar gran diferencia entre los andaluces, que son los más insatisfechos al respecto (para el 44\% éste es insuficiente) y los no andaluces, de los que sólo el $17^{\prime} 6 \%$ lo ve insuficiente, siendo suficiente para el 70'6\% de ellos frente al 38'5\% de los andaluces que opina esto mismo. Por otra parte, se muestran más satisfechos con el asunto los de Primaria: el $45^{\prime} 4 \%$ lo ve suficiente, mientras que esto mismo opina el 34'5\% de los de Secundaria.

La imagen de Andalucía en los libros de texto es pobre o escasa para el 30'3\% de los andaluces y para el $17^{\prime} 6 \%$ de los no andaluces, mientras que es positiva o buena para el $29^{\prime} 4 \%$ y el $47^{\prime} 1 \%$ respectivamente. Hay otro contraste notable, aunque poco significativo numéricamente: esa imagen es excesivamente nacionalista para el 0 '9\% de los andaluces y para el 11'8\% de los no andaluces. Por ciclos, la consideran más pobre los de Secundaria (34'5\%) que los de Primaria (26'8\%).

En cuanto a la formación reciente sobre cultura andaluza, el 23 ' $1 \%$ de los andaluces que no han asistido en los últimos tres años a actividades de este tipo aducen que se debe a que han sido pocas las actividades ofrecidas, cosa que sólo es así para el 7’7\% de los no andaluces que no han realizado tal tipo de actividades de formación.

Andalucía está presente en sus clases con temas o actividades específicas para el $53 \%$ de los no andaluces y para el $36,7 \%$ de los andaluces, y regular, poco o nada presente para el $17^{\prime} 7 \%$ y el $33^{\prime} 9 \%$ respectivamente. Este dato podemos interpretarlo de dos formas que son casi opuestas: o bien una mayoría de no andaluces, en efecto, prestan más atención a Andalucía en sus clases, o bien es una cuestión de percepción, es decir, consideran que la tratan mucho en clase independientemente de que realmente sea así o no. Nos orientamos más por esta segunda interpretación, que está más acorde con la mayoría del resto de respuestas de los no andaluces. Por niveles, no es grande el contraste, salvo que está poco o nada presente para el 21'1\% de los de Primaria y para el 34'5\% de los de Secundaria.

Declaran que en su clase hay símbolos de España el 41 '2\% de los no andaluces y el 21 ' $1 \%$ de los andaluces. En cuanto a símbolos sobre las demás entidades, no hay contrastes significativos entre ambos grupos. Esta diferencia puede ser, otra vez, muy relativizable, también por la percepción del asunto. No tenemos la seguridad, por ejemplo, de que todos los que tienen en clase un retrato del Rey lo

no son, en ningún caso, maestros sino "profesores de instituto». Esta separación funciona claramente, a nivel intemo, entre los propios docentes, y da lugar a relaciones, actitudes, estereotipos y percepciones que merecerían ser observadas con detenimiento. 
hayan considerado como lo que es, como un símbolo de España; o de que, por ejemplo, un cartel de la Junta de Andalucía sobre el medio ambiente, salud o cualquier otro tema sea considerado por todos como un símbolo de Andalucía ${ }^{8}$. En esto hay notables diferencias entre los de Primaria y los de Secundaria. Los primeros tienen símbolos de Andalucía en el 35\% de los casos, por sólo el 17'2\% de los de Secundaria, y de la localidad en el $23^{\prime} 7 \%$ y el 6'9\% de los casos respectivamente. Por el contrario, el $20{ }^{\prime} 7 \%$ de los de Secundaria tienen en clase símbolos de Europa, por sólo el 10 '3\% de los de Primaria.

Para el 55' $1 \%$ de los andaluces son necesarios símbolos de Andalucía en clase, frente al $42{ }^{\prime} 9 \%$ de los no andaluces que opinan así. De la localidad, los consideran necesarios el 42 ' $2 \%$ de los andaluces y el $29^{\prime} 4 \%$ de los no andaluces. De España, y aquí hallamos otra considerable diferencia, el $35^{\prime} 8 \%$ y el $50 \%$ respectivamente. Por niveles, los de Secundaria ven necesarios símbolos de Europa en el $41^{\prime} 4 \%$ de los casos, frente al $27^{\prime} 8 \%$ de Primaria. Los de Primaria ven necesarios símbolos de España en mayor proporción (40’2\%) que los de Secundaria (24'1\%), y lo mismo ocurre respecto a la localidad (46'4\% y 20 '7\% respectivamente).

Se les pidió a los docentes, también, indicar por orden de preferencia (de 1 a 4, siendo 1 la puntuación para la más preferente) el nivel de identificación que debería fomentar la escuela. Éste es el andaluz (cuya puntuación media es de 1'96), con notable ventaja respecto al local (2'3), al español (2’31) y al que queda muy atrás en las preferencias, el europeo ( $\left.3^{\prime} 13\right)$. Son los de Primaria los que otorgan mayor preeminencia al andaluz (1'9), puesto que los de Secundaria eligen como opción preferente la identificación española (1'92). A la identificación andaluza sigue, para los de Primaria, la local (2'06), la española (2'42) y por último la europea (3’26). Para los de Secundaria, a la española le sigue la andaluza (2'15), posteriormente la europea ( $\left.3^{\prime} 08\right)$, y en último lugar la local (3'12). La diferencia entre ambos colectivos ha resultado, pues, muy notable. Teniendo en cuenta el lugar de nacimiento, los andaluces optan por la andaluza ( 1 '96), y después por la local (2’25), la española (2’33) y la europea ( $\left.3^{\prime} 14\right)$; los no andaluces, por la andaluza (en la misma medida casi que los anteriores: 1'93), seguida por la española (2'19), la local (2'6) y, por fin, la europea (3'07).

Parece haber una tendencia mayoritaria, por tanto, hacia la preferencia por el fomento de la identificación andaluza, y luego la local o la española. Sin embargo, estos datos adquieren su verdadero sentido si se los compara con el nivel de identificación que, en su opinión, realmente fomenta la escuela. Contabilizando las puntuaciones, observamos que hay un notable desfase, precisamente, para el caso de la identificación europea: ésta recibe 29 puntos más (utilizamos el mismo cri-

8. Iucluso pudiera no ser considerado como un símbolo de Andalucía, y con toda razón, un cartel de la Junta conteniendo el logotipo que ha sustituido recientemente al escudo, en un nuevo paso hacia la eliminación de elementos que puedan constituir señas de identidad andaluza. 
terio de puntuación que para el caso anterior) como identidad realmente fomenta. da que deseada, por lo que a juicio de los docentes esta identificación se foment menos aún de lo que sería deseable. Esto ocurre más entre los de Primaria (1 puntos) que entre los de Secundaria (10 puntos), y más entre los andaluces (21 puntos) que entre los no andaluces (8 puntos). Podríamos deducir de aquí que aunque el fomento de la identidad europea no es precisamente el prioritario parc los docentes, entienden que la escuela lo fomenta demasiado poco, lo que indice una creciente sensibilización y predisposición favorable hacia esto. Algo se mueve. En cambio, la andaluza recibe sólo un punto menos como identificación deseada que como realmente fomentada, lo que muestra un grado de acuerdo notable con lo que realiza la escuela en este sentido. Aquí hay no obstante diferencias marcadas: es fomentado en demasía (4 puntos) para los de Secundaria, y justo lc contrario para los de Primaria (5 puntos). Para los andaluces ( 2 puntos) es algc menos fomentado de lo deseable, mientras que para los no andaluces ocurre, aunque mínimamente ( 1 punto), lo contrario. La local es más fomentada que deseada para los de Primaria (11 puntos), mientras que hay correspondencia plena eutre preferencias y realidad para los de Secundaria. En el caso de los andaluces, es más fomentada realmente que deseada ( 7 puntos) y lo mismo, aunque en menor medida, para los no andaluces (4 puntos).

Por último, el desfase entre preferencia y realidad es de 8 puntos en la identificación española: para los de Primaria ésta es más fomentada que deseada ( 8 puntos), mientras que para los de Secundaria hay, como en el caso anterior, una diferencia 0 . Lo mismo ocurre según el lugar de nacimiento: 8 puntos en el caso de los andaluces, 0 en el de los no andaluces.

En algunos casos han respondido otros niveles o modelos de identificación: "ciudadano del mundo» (6 veces), "universalismo» (4 veces), "humanidad», "persona» o "tolerancia» (8 veces), e «igualdad» o "solidaridad» (4 veces), lo que suma un 17 ' $5 \%$ de respuestas de este tipo. También se ha respondido la «familia» (2 veces) y el «barrio» (1 vez).

De todas formas, en este asunto nos quedamos preferentemente con los datos referentes a la identificación europea, que por expresar la mayor diferencia entre lo deseado y lo que se entiende como más fomentado realmente, pueden ser más significativos.

Por otra parte, el $82 ` 4 \%$ de los no andaluces organizó cosas con motivo del Día de Andalucía, más que los andaluces (64’2\%); y mucho más los de Primaria (el $78^{\prime} 3 \%$ ) que los de Secundaria (el 24'2\%).

La materia impartida se debe enfocar igual aquí que en Madrid para el 62'1\% de los de Secundaria y para el 30'9\% de los de Primaria, teniendo un peso decisivo en esto cuál es la «asignatura» que dan.

Fomentar un sentido nacionalista andaluz se presenta como deseable para el 48'5\% de los de Primaria, mucho más que para los de Secundaria (el 17’2\%). Para 
éstos, además, es un asunto más indiferente (34'5\%) que para los de Primaria (9'3\%). Interesante contraste, que en cambio no hallamos en alto grado entre andaluces (deseable para el $42^{\prime} 2 \%$, no deseable para el $39^{\prime} 4 \%$ e indiferente para el $13{ }^{\prime} 8 \%$ ) y no andaluces $\left(35^{\prime} 3 \%, 41^{\prime} 2 \%\right.$ y $23^{\prime} 5 \%$ respectivamente).

El acontecimiento histórico más positivo es el descubrimiento y comercio con América para el $177^{\prime} 6 \%$ de los no andaluces (por el 5'5\% de los andaluces), y la Expo'92 para el 11 ' $8 \%$ y el 2' $8 \%$ respectivamente. En total, los acontecimientos con papel subsidiario de Andalucía (Expo y AVE, descubrimiento de América y democracia) son lo más positivo para el 41 '1 \% de los no andaluces y para el $13{ }^{\prime} 8 \%$ de los andaluces. También se da en esto una clara diferencia según nivel impartido: el 34'5\% de los de Secundaria estima este tipo de acontecimientos como los más positivos para Andalucía, frente al 12'4\% de los de Primaria.

En cuanto al acontecimiento más negativo, los mayores contrastes son éstos: mientras éste consiste en hechos relacionados con la inserción de Andalucía en redes más amplias (Unión Europea, OCM del aceite, Expo y especialización en servicios) para el 8 '2\% de docentes andaluces, ningún no andaluz señala cuestiones de este tipo. Por el contrario, determinados aspectos de la política autonómica del PSOE son señalados por el $11^{\prime} 8 \%$ de los no andaluces y sólo por el 0 ' $9 \%$ de los andaluces. Por último, la «Reconquista» y hechos relacionados con la misma y sus consecuencias constituyen lo más negativo para el $14^{\prime} 7 \%$ de los andaluces y para el 5'9\% de los no andaluces.

En suma, si hacemos una jerarquía de contrastes, observamos una enorme diferencia entre andaluces y no andaluces (de más de 20 puntos) en la valoración sobre el peso de Andalucía en el currículo y en los libros de texto, que consideran justo y suficiente los segundos, y escaso o insuficiente los primeros. También hay una gran diferencia en la consideración de los acontecimientos históricos, en los que los no andaluces ponen el peso en episodios en los que Andalucía juega un papel subsidiario o pasivamente integrado en España.

En segundo lugar, hay grandes diferencias (de 15 a 20 puntos) en la consideración de la imagen que los libros de texto ofrecen sobre Andalucía, que es mejor valorada por los no andaluces; en la organización de actividades con motivo del 28-F (los no andaluces las han organizado en mayor proporción); y en la presencia de Andalucía en clase, que es mayor (aunque con los matices apuntados) para los no andaluces.

Por último, hay diferencias notables (entre 10 y 15 puntos) en otros aspectos Ia imagen de los libros de texto es entendida como más pobre para los andaluces (y más excesivamente etnonacionalista para los no andaluces), las actividades de formación sobre cultura andaluza son pocas para los andaluces en mayor proporción que para los no andaluces, los símbolos en clase han de ser, en bastante mayor proporción para los andaluces, de Andalucía y de la localidad (mientras que de España en mayor medida para los no andaluces), la indiferencia hacia la conve- 
niencia de fomentar en la escuela un sentido nacionalista andaluz es mayor entre los no andaluces, y también hallamos notables contrastes en algunos aspectos acerca de la valoración de los hechos históricos, ya señalados.

Si comparamos la opinión de los docentes de Primaria y Secundaria, la diferencia es muy grande (más de 20 puntos) en la realización de actividades en clase con motivo del 28-F, que han hecho en mayor proporción los de Primaria (más de 50 puntos por encima); en la consideración de que fomentar un sentimiento nacionalista andaluz es deseable (eso opinan mucho más los de Primaria), en la indiferencia al respecto (mucho mayor en los de Secundaria), y en la consideración de los acontecimientos históricos positivos en los que Andalucía juega un papel subsidiario (los de Secundaria).

La diferencia es grande (entre 15 y 20 puntos) en la presencia de símbolos de Andalucía y de la localidad en clase, y en la necesidad de que haya símbolos de España y de la localidad en la misma, que en todos los casos es considerablemente mayor entre los docentes de Primaria.

Por último, hay contrastes notables (de 10 a 15 puntos) en cuanto al organismo al que acuden para realizar actividades, pues el Ayuntamiento es claramente más utilizado en Primaria; en la presencia de Andalucía en clase, que es más escasa o inexistente en Secundaria; en la valoración de los libros de texto, que para los de Primaria ofrecen suficiente espacio a Andalucía; y en la presencia de símbolos de Europa en clase, así como en la consideración de su necesidad, que son mayores en Secundaria.

De estos datos no nos atrevemos a sacar conclusiones, puesto que esto sólo debe hacerse con una muestra mayor, con unos criterios de representatividad previamente establecidos y con un cuestionario realizado con criterio mucho más «sociológico», y aún así hay que ser muy precavidos. Sólo hemos querido poder intuir tendencias generales y actitudes concretas de los docentes ante un cuestionario de tema (y a veces corte) etnonacionalista. Expuestos están los datos generales ${ }^{9}$. Ha habido reacciones muy diversas, aunque éstas se relacionan por lo común con el grado de afinidad hacia el compañero que les ha presentado el cuestionario ${ }^{10}$. Normalmente, si su relación con el que pasa el cuestionario es buena, se lo han devuelto relleno; si, por el contrario, es tensa o fría, no lo han rellenado. Ha habido algunos comentarios críticos sobre el mismo (aunque no muchos, ciertamente: esperábamos más) por su enfoque excesivamente «localista», o por la complejidad del tema y el carácter excesivamente cerrado o excesivamente abierto de algunas preguntas. Casi el $50 \%$ de los cuestionarios entregados han sido rellena-

9. Podemos hacer desagregaciones y considerar otras variables y relaciones, pero preferimos no hacerlo con una muestra de sólo 126 encuestados.

10. En muchos casos el cuestionario no lo ha pasado el investigador, sino un docente del centro encuestión. Agradecemos a éstos su colaboración, que no ha consistido sólo en pasar el cuestionario sino también en informarnos acerca de las reacciones $y$ visicitudes relacionadas con el mismo. 
dos; el resto, no recepcionados o no devueltos. En algunos casos ha provocado interés (especialmente entre algunos docentes de Primaria), y en otros (en especial de Secundaria) rechazo o absoluto desinterés.

Los docentes, en lo referente a Andalucía en la escuela, mantienen posturas diversas pero, en general, no muy articuladas, al menos en lo que concierne al centro educativo entendido como totalidad. Un aspecto expresivo es la consideración de que las oposiciones a la enseñanza en Andalucía deben ser sólo para andaluces o vinculados a Andalucía para el 9'5 \% de los docentes encuestados; la gran mayoría considera que tienen que ser abiertas: el 39'7 \% abiertas sin más, y el 50 '8 \% con un temario específico sobre Andalucía. De todos modos, este último dato indica que en esto van por delante de la propia Administración educativa andaluza. Hay diferencias en esta apreciación según el lugar de nacimiento, puesto que opina que las oposiciones han de ser abiertas a todos sin más condición un $76^{\prime} 4 \%$ de los no andaluces y un 33'9\% de los andaluces (una diferencia enorme, de más de 40 puntos); y abierta a todos pero con temario sobre Andalucía el 17' 6 $\%$ y el $56 \%$ respectivamente (casi 40 puntos de diferencia). También se observan contrastes según el nivel: han de ser abiertas a todos sin condición para el $34 \%$ de los de Primaria y para el 58'6 \% de los de Secundaria, y abiertas pero con temario específico para el 54' $6 \%$ y el 37 '9 \% respectivamente.

En Secundaria tiene gran peso la materia que el docente imparte, por encima de otras consideraciones, y ésta se entiende a menudo de manera pretendidamente aséptica: "Amo mi tierra, pero mi materia es otra" (profesor de Secundaria). En Primaria, por el contrario, el alumno adquiere centralidad, como también la localidad posee mucho mayor relieve. En ambos casos, esto es por influencia del propio plan de estudios, pero no hay que despreciar otros componentes. A veces se repiten estereotipos que parecen bien asumidos: "La cultura andaluza es universal y traspasa los límites de lo puramente nacionalista» (profesor de Secundaria). El nacionalismo se identifica a veces con lo localista empobrecedor: "Se le está dando mucha importancia a los nacionalismos y es más interesante que nos demos cuenta de que somos personas antes que nada» (de Primaria); "Localista, cae en el aldeanismo", nos advierte otro de Primaria. A veces, además, se lo relaciona con la insolidaridad y con la exclusión: "El nacionalismo es excluyente y limitado, y fomenta la insolidaridad e intolerancia" (de Secundaria); "Que conozcan su cultura, pero sin olvidar la solidaridad con otras culturas y el respeto» (de Primaria); "Es necesario un esfuerzo de los docentes para defender las senas de identidad dentro de la unidad pero respetando la diversidad» (de Primaria). A veces, se asocia a los excesos: «Es bueno fomentar el amor a su tierra, pero no los nacionalismos demasiado exagerados» (de Primaria). Algunos son reacios a los símbolos etnonacionalistas: "No me hacen falta símbolos ni otras cosas para amar mi tierra» (de Primaria); "No hay que hacer de lo propio bandera de nada, si acaso de universalidad» (de Secundaria). Esa universalidad puede estar tan asumida, para 
el caso andaluz, y se relaciona tanto con sentimientos no etnonacionalistas, que en la misma base de lo andaluz está la imposibilidad del nacionalismo: "Es dificil plantear una enseñanza localista cuando tendemos a abrirnos a otros pueblos» (de Primaria). Se dan también voces de queja, en el sentido de que "No valoramos suficientemente lo nuestro" (de Primaria), o de que "sólo hay una asignatura sobre nuestro patrimonio cultural, y encima optativa» (de Primaria; se refiere a «Patrimonio Cultural de Andalucía»).

En todo caso, y más allá de lo que deducimos de las respuestas a este cuestionario y de las opiniones de los docentes, es preciso tener claro que la escuela proporciona un contexto complejo, un contexto que trasciende los contenidos que se transmiten-adquieren en la misma, puesto que las formas de hacerlo, las interacciones formales e informales, los rituales escolares, los símbolos presentes, el juego de pugnas y alianzas entre sus principales actores (docentes, alumnos, padres y Administración, sobre todo), etc., la dotan de una extraordinaria riqueza que, muy a menudo, no es percibida, especialmente en los estudios que se hacen desde «dentro» ${ }^{11}$.

\section{LA VISIÓN DE LOS ALUMNOS: UNA PEQUEÑA REDACCIÓN}

En cuanto a los alumnos, se trata de una categoría social, que éstos van asumiendo progresivamente, que los define por su edad, por su necesidad de integración a un sistema más amplio que el constituido por las redes de parentesco y vecindad (siendo entrenados para la incorporación al sistema como productoresconsumidores y como «ciudadanos nacionales»), y por sus carencias y necesidad de normalización. Son objeto de transmisión de materias curriculares arbitrarias, de habilidades y de ideologías. En la escuela, el niño aprende hechos y disposiciones sobre (en lo que aquí nos interesa) los grupos etnonacionales, en un proceso que es a la vez cognitivo y afectivo. Reglas de comportamiento, valores y nociones son los elementos con los que construye su representación del mundo social, lo que incluye la adquisición de estereotipos, así como la asunción de los distintos grados de pertenencia ${ }^{12}$. Dentro de este proceso hay un punto que nos resulta de gran interés, y que se apunta desde la sociología política: la importancia del fracaso escolar sobre la autovaloración y expectativas de los niños, que ante esto, a menudo asumen la sumisión y la incompetencia política. La comparación de los índices de fracaso escolar entre diversas Comunidades Autónomas puede ser de gran significación, si buscamos sus implicaciones etnonacionales y políticas.

11. Y que muchas veces incorporan de manera totalmente inadecuada el término «etnografía» en sus páginas. Una admonición contra esto es, en buena medida, la ilustratia obra de H. Velasco y A. Díaz de Rada (1997).

12. Para un enfoque psicológico del tema, cfr. Delval (1982), Phinney y Rotheram (1987) y, sobre todo, Delval (1989). 
Recientemente se han publicado en la prensa (marzo de 1998) los resultados de un estudio encargado por el Ministerio de Educación y Cultura, que pretende evaluar especialmente el nivel de los alumnos de ESO. Andalucía no ha participado, en parte por desavenencias partidistas, en parte por temor del PSOE a los resultados (creemos). Según este estudio, los alumnos de Cataluña tienen un especialmente bajo nivel de instrucción. La respuesta del Gobierno catalán no se hizo esperar: la población inmigrada es responsable de este bajo rendimiento. El estudio de casos o incidentes, como éste, puede ser enormemente revelador.

Por nuestra parte, hemos hecho una pequeña prueba, en este caso redacciones sobre el tema «Andalucía» por parte de alumnos de un grupo de 17 alumnos (los presentes ese día) que cursan $2^{\circ}$ de ESO en un instituto de un barrio periférico de Sevilla. No vamos a detenernos en el contexto, ni en las situaciones o en el medio empleado para obtener información (la realización de un escrito), aspectos cuya consideración es imprescindible, porque igualmente sólo pretendemos trazar una instantánea que nos permita poder apuntar sugerencias para un estudio más detallado.

Sugerido el tema en clase, así en general, los alumnos pidieron al profesor que les ofreciera pautas o un guión, pues no se les ocurría qué escribir, lo que ya es bastante expresivo. El profesor, entonces, espontáneamente, les indicó que podían hablar de lo siguiente: presentación (localización y provincias), características geográficas (relieve, ríos, clima, parques naturales), población, economía y trabajo, gobiemo, rasgos culturales (ejemplificó: religión, habla, gastronomía), cómo somos, monumentos y fiestas. Los alumnos siguieron este guión, sin añadir temas no planteados aquí, aunque saltándose otros sí planteados.

En la presentación, Andalucía es calificada mayoritariamente como Comunidad Autónoma (por 9 de los 17 alumnos), y 6 no nombran en ningún momento su estatus político. Casi todos (16) señalan sus provincias, y 3 indican la capitalidad de Sevilla. Uno nombra Andalucía como «una de las provincias más grandes y pobladas» ${ }^{13}$, y otro como "la ciudad que está al sur de España». Para otro, «mi comunidad es la mejor de todas».

Sobre sus características físicas, más de la mitad (9) indican que el relieve es llano o poco montañoso, lo que expresa que no hay una percepción de Andalucía como totalidad. Esta impresión se acentúa si vemos la semblanza que trazan algunos del río Guadalquivir: "el Guadalquivir nace en Huelva, recorre toda Huelva, toda Sevilla y llega hasta Córdoba»; "Sevilla tiene un río especial que es el Guadalquivir»; "hay un río muy importante que pasa por Sevilla. Ese rio es el Guadalquivir»; "de Cádiz el río Guadalquivir, en Sevilla el Guadalquivir, en Córdoba el Genil». Aparte de estas cuatro referencias, hay otras nueve en las que sí se relaciona este río con Andalucía en general o con buena parte de la misma, aun-

13. En la transcripción, corregimos las faltas de ortografia, muy abundantes. 
que en uno de estos casos sus principales afluentes resultan ser el Tinto y el Odiel Se citan, además del Guadalquivir, estos dos ríos (por parte de 3 alumnos) y, sólc en un caso cada vez, el Genil, el Guadalete y el Guadiana. La percepción que tie. nen estos alumnos es, básicamente, la de la baja Andalucía. Se nombra por parte de la mayoría Doñana, aunque sólo uno hace referencia al desastre ecológico pro. ducido dos días antes de la elaboración del escrito; uno de ellos ubica Doñana er Sevilla.

Sólo 5 se atreven a decir el número de habitantes: según 3 de ellos, somos $\varepsilon$ millones; para otro, somos 6 millones, y según otro, 9. Hay quien hace una aventurada incursión en busca de elementos causales: "Somos muchísimos habitante: por $\mathrm{km}$. cuadrado, por lo que al no haber trabajo la gente se tiene que dedicar c robar o a atracar bancos, $u$ otras actividades que rompen la ley».

El trabajo se relaciona sobre todo con la industria. Para 6 es así, mientras que las empresas comerciales y tiendas, la agricultura y la ganadería, y la carpintería aparecen citados, respectivamente, en una ocasión. La industria andaluza se califica como poco desarrollada o escasa en 5 casos. Cuando hablan de «riquezas» en 9 casos se nombra el olivo o el aceite, en 5 la vid o el vino, en 3 las naranjas y los cereales, y en uno las playas y el turismo. Parece percibirse la riqueza económica en términos de producción agrícola, y el pregonado papel del turismo nc ha calado entre estos alumnos o lo conceptúan en un campo distinto del de «riqueza». Hay una voz algo crítica (aunque vaga) con la OCM del aceite: "el aceite abunda mucho pero hace un tiempecito que se lleva muy malamente». El tema que más se repite al hablar sobre la economía y el trabajo en Andalucía es el del parc (11 hablan de él), pero sólo en un caso (aparte del mencionado arriba) se buscan sus causas, también con gran desorientación por cierto: "La población en Andalucía ha aumentado, por lo que buscar trabajo es muy dificil». Se inventan cifras. lo que nos muestra el valor más cualitativo y afectivo que éstas tienen para los alumnos y su noción acerca de lo empírico: según uno, "el 60\% de las personas está cobrando el paro»; para otro, hay 3 millones de parados; otro considera que el 50 por ciento de los andaluces está parado, y otro que «casi la mitad de Andalucía está parada».

De los temas propuestos por el profesor, el que menos ha sido citado es el referente al gobierno. Se trata de un asunto, al parecer, especialmente ignorado y poco atractivo. Tan sólo 3 hacen referencia al mismo. Uno lo único que dice es que "tenemos un gobierno electivo", otro que "El gobierno es autónomo, el presidente es Manuel Chaves y es del partido socialista», y el tercero señala: "Respecto al gobierno, cada provincia tiene un alcalde pero el presidente es el que gobierna, que manda en toda España». El nivel de conocimiento en esto está bajo mínimos, y la confusión conceptual es grande. Por otra parte, hay una referencia al Día de Andalucía: «El día de Andalucía se celebra el 28 de Febrero y se hacen carreras y juegos para todos». 
Sobre la religión de los andaluces, 7 señalan algo. Para uno, «la religión es cristiana y somos monoteístas»; para otro "su religión es cristiana». Los demás entran en valoraciones desiguales: "somos muy religiosos y la mayoría somos cristianos, exceptuando algunos que son de otras religiones como mormones, ateos, testigos de Jehová, etc.»; "la mitad de la población de los andaluces son muy religiosos». Otro considera que "somos poco religiosos y muy duros», y para otro "Al contrario de los demás países aquí no le damos mucha importancia a la religión». El último, por fin, parece ubicar mejor el asunto: «Los andaluces cada vez somos menos religiosos, sin embargo nos gusta mucho la Semana Santa».

Sobre la gastronomía, otros 7 comentan algo. A veces con juicios de valor positivos, y a veces sólo de manera enunciativa. Se nombra sobre todo el gazpacho, y luego, una sola vez para cada caso, los chícharos, pucheros, pescaíto frito, langostinos, paella, aceite, torrijas y lechuga.

El habla merece la atención de 5, en estos términos: «El habla sobre todo es burra»; "Hablando somos un poquito burros"; "Lo malo de los andaluces es que hablamos muy malamente porque nos comemos muchas palabras y no las pronunciamos»; "A veces somos muy horteros hablando, pero otras muy educados»; y "Los andaluces son bastante bastos hablando». No sabemos qué opinarán los que no han referido nada del asunto, pero el panorama parece bastante desolador en este sentido. Deben ser grandes consumidores de televisión, y la escuela parece no tener peso aquí, y si lo tiene no es precisamente en el sentido de apreciar las propias formas de expresión.

Señalan que Andalucía tiene muchos monumentos, que "sus mejores riquezas son los monumentos históricos» y que "sus monumentos son muy famosos». Suelen poner una lista de los mismos y su localización. El más citado es la Giralda (9 veces), y luego la Mezquita de Córdoba (8), la Alhambra (7), la Torre del Oro (6), la Catedral de Sevilla (4) y el Alcázar de Sevilla (2). Es decir, 4 monumentos sevillanos entre los 6 más destacados.

Sobre las fiestas se dice que en Andalucía hay muchas, y que son «muy famosas», «muy bonitas y muy alegres», «muy divertidas»; se señalan por lo común las fiestas "más conocidas», que resultan ser la Feria de Sevilla (10 veces), la Semana Santa de Sevilla (8), los camavales de Cádiz (6), la Feria en singular y sin nombrar de dónde (5), la Semana Santa en las mismas condiciones que la anterior (5), los carnavales (4), el Rocío (2) y las colombinas de Huelva (2). Sevilla aparece en este apartado casi omnipresente, seguida de Cádiz y Huelva.

Hallamos 6 semblanzas de cómo somos: «Somos muy alegres y simpáticos, pero sobre todo los de Sevilla somos los más alegres, simpáticos y nos gusta mucho una juerga». Esta visión alienante, vieja conocida, no se ha desterrado, sino que cobra auge hoy. "Somos muchos y la mayoría simpáticos y un poco burros, sobre todo en Cádiz, Huelva y Sevilla»; «Los andaluces somos muy salerosos. A los extranjeros les gustan mucho las sevillanas (baile)»; "La mayoría de la gente es simpá- 
tica y se porta bien con los demás». Podremos reconocer esto en algunos de lo: aspectos de la propia imagen de Andalucía que dan los libros de texto. Otro alumnc recurre, aunque con sentido exculpatorio, a la imagen externa: "Los de Andalucíc tenemos fama de flojos y poco trabajadores, aunque flojos y poco trabajadores ha) en todas partes». Por último, para dos de ellos, "Somos personas normales», k que no deja de producir cierto alivio en vista de la anterior autoimagen, absoluta. mente mediatizada y alienada, que además podría provocar perplejidad después d más de quince años de autonomía si no fuera porque conocemos cómo se vient desarrollando la misma.

Hay, por otra parte, valoraciones sobre ciertos aspectos de Andalucía: «Es lc Comunidad Autónoma más bonita, no porque viva allí, sino por la cantidad de monumentos, fauna, flora, historia, etc. que posee». Los monumentos, como las fiestas y la gastronomía, gozan del favor general, y se convierten así, en el casc de los dos primeros, en signos con los que hay una identificación positiva, al con. trario de lo que ocurre con el habla. Muchos demuestran una falta absoluta de visión integrada sobre Andalucía, pero expresan los problemas que viver cotidianamente: "Andalucía está un poco sucia»; "Cada vez hay más violencia er las calles de Andalucía». Algunos resaltan, como hemos visto, la importancia vi. tal de los otros para valorar Andalucía: "Al cabo del año vienen muchos extranje. ros, sobre todo a Sevilla»; "Es la Comunidad Autónoma que más se conoce y que más fama tiene».

Las conclusiones a las que podemos llegar analizando estos escritos son, especialmente, las siguientes:

- El conocimiento sobre Andalucía es considerablemente escaso, fragmentario, y adolece de una gran confusión conceptual.

- No hay una clara visión integrada de la misma, y se tiene más percepción indiscutiblemente (viven en Sevilla), de la baja Andalucía.

- El localismo es feroz, llegando a veces a un extremado chovinismo, comc vemos en alguna de las citas precedentes o en ésta: «Sevilla es nuestra ciudad, o sea la mejor de todas las ciudades de Andalucía». Esto se expresc también en la propia articulación de los textos, que se estructuran por provincias, y en la insistencia en que cada ciudad tiene sus rasgos propios y diferenciados o en que todas las ciudades son muy distintas.

- La visión y valoración que se tiene es altamente dependiente de la que desde el exterior se hace.

- Se asumen e interiorizan los tópicos sobre nuestro «carácter» y nuestro habla, con una alarmante falta de sentido crítico.

Estos alumnos nacieron al mismo tiempo, o muy poco después, que la autonomía. No han conocido otra realidad política. ¿Qué se ha hecho para incidir en la 
conciencia de los andaluces de este período? Por otra parte, estos alumnos han seguido el nuevo sistema LOGSE, valorado muy positivamente por la Consejería de Educación a partir de un estudio que comparaba con los alumnos del sistema anterior. No podemos generalizar con una muestra de 17 casos, pero el leve surco al que nos hemos acercado para mirar más de cerca, aunque sea insignificante en un campo extensísimo, nos deja francamente preocupados.

\section{ANEXO}

Como hemos indicado, el cuestionario se ha realizado con poco «espíritu» de encuesta y con un propósito meramente auxiliar, complementario de nuestro centro de interés, que lo conforman las disposiciones legislativas, los libros de texto y discursos y medidas políticas. Los objetivos generales del mismo han sido:

- Realizar una «cata» de la escuela como fuente directa.

- Conocer las respuestas a las cuestiones planteadas, como complemento de nuestro tema.

- Controlar, en relación con el objetivo anterior, algunas variables por hallar correlaciones o tendencias significativas.

- Recibir impresiones sobre la reacción ante el tema y el propio cuestionario, planteado con pocos disimulos.

Hemos comentado ya lo más relevante acerca de estos puntos, excepto del primero. Es destacable, con respecto a éste, el hecho de que la escuela, en contra de la impresión que quizás pudiera tenerse en principio, es un escenario al que, como ocurre con otras instituciones, hay que acceder muy poco a poco y pisando con muy especial cuidado. Es difícil penetrar en ella, tanto por la cantidad de códigos no explícitos con los que es preciso familiarizarse, como por su propio ritmo y dinámica muy densos en significaciones y absorbentes para sus agentes; también porque el nivel de prevenciones y susceptibilidades es ciertamente muy alto.

Han sido 250, aproximadamente, los cuestionarios entregados. De los 14 centros docentes en los que se ha pasado, en 4 lo hemos hecho personalmente, y en el resto lo hicieron docentes de los mismos que tuvieron a bien colaborar, tanto en esto como en contamos las incidencias habidas. Llegaron finalmente a nuestras manos 128 cuestionarios (muy poco más de la mitad de los entregados), de los que tuvimos que rechazar 2 por diversos motivos, de modo que la muestra ha sido de 126 docentes. De éstos, 97 son de Primaria y 29 de Secundaria (menos proclives han sido éstos a responder el cuestionario). Por otra parte, 109 de los docentes son nacidos en Andalucía y 17 en otros lugares del estado español. El escaso número de los nacidos fuera de Andalucía nos hace ver con especial dis- 
tancia las informaciones recogidas a partir de esta variable que, insistimos, son er cualquier caso llamativas ${ }^{14}$. Por otra parte, todas son cantidades muy reducidas como para sacar conclusiones tajantes de los datos que hemos ofrecido, y por estc conviene relativizar los resultados aunque tampoco hay, desde luego, que renunciar a priori a ellos.

A continuación, exponemos las contestaciones a algunas de las cuestiones más significativas a las que nos hemos referido. Ofrecemos sólo un extracto del cuestionario, que incluyó también preguntas más abiertas cuyas respuestas no podemos incluir aquí. Las respuestas las ofrecemos desagregadas según las dos variables que hemos encontrado significativas y que como tales hemos tenido en cuenta ${ }^{15}$ : el nive] de enseñanza impartido y la Comunidad donde han nacido ${ }^{16}$.

- ¿Qué peso tiene Andalucía en el currículo? (Contestar con una X a la izquierda de la respuesta que le parezca más apropiada, tanto aquí como enlas demás cuestiones, salvo indicación contraria).

$\begin{array}{llllll}\text { Demasiado } & \mathrm{P}=2 & \mathrm{~S}=0 & \mathrm{~A}=1 \quad \mathrm{~B}=1 & \mathrm{~T}=2 \\ \text { E1 justo } & \mathrm{P}=45 & \mathrm{~S}=15 & \mathrm{~A}=48 & \mathrm{~B}=12 & \mathrm{~T}=60 \\ \text { Poco } & \mathrm{P}=42 & \mathrm{~S}=12 & \mathrm{~A}=32 & \mathrm{~B}=2 & \mathrm{~T}=54 \\ \text { Ninguno } & \mathrm{P}=0 & \mathrm{~S}=2 & \mathrm{~A}=1 \quad \mathrm{~B}=1 & \mathrm{~T}=2 \\ \text { N/C } & \mathrm{P}=8 & \mathrm{~S}=0 & \mathrm{~A}=7 \quad \mathrm{~B}=1 & \mathrm{~T}=8\end{array}$

\section{- ¿Organizó algo a nivel de clase con motivo del pasado 28-F?}

$\begin{array}{llll}\text { No } & \mathrm{P}=18 \quad \mathrm{~S}=20 & \mathrm{~A}=35 \mathrm{~B}=3 & \mathrm{~T}=38 \\ \text { Sí (especificar) } & \mathrm{P}=76 \quad \mathrm{~S}=8 & \mathrm{~A}=70 \quad \mathrm{~B}=14 & \mathrm{~T}=84 \\ \text { N/C } & \mathrm{P}=3 \quad \mathrm{~S}=1 & \mathrm{~A}=4 \quad \mathrm{~B}=0 & \mathrm{~T}=4\end{array}$

14. No ha de entenderse que nuestra posición es contiene juicios contrarios a que accedan a la docencia en Andalucía profesionales de otros lugares. Nos hemos limitado a exponer datos. Por otra parte, conocemos docentes no andaluces con clara conciencia respecto a la singularidad de Andalucía y con compromiso hacia el desarrollo de su identidad étnica. Como ejemplo, un caso del que tuvimos noticia: una profesora de Primaria, no andaluza, aprovechó una reunión con los padres para insistirles en que fomentaran y valoraran positivamente el habla andaluza, pues notaba actitudes de poca estima, e incluso complejo, en muchos de sus alumnos sobre su propia forma de hablar.

15. Otras variables que hemos visto, y desechado por poco significativas debido a lo exiguo de la meustra o/ y por falta de relevancia son: localidad de nacimiento, localidad del centro educativo, cursos impartidos, materias impartidas y realización reciente o no de cursos de formación sobre cultura andaluza. Por otra parte, los cruces de variables los rechazamos por la escasa cantidad de encuestados.

16. Respectivamente: en cuanto al nivel impartido, P (Primaria y/o Primer Ciclo de Secundaria) y S (Secundaria completa o Segundo Ciclo de Secundaria); y en cuanto al nacimiento, A (andaluces) o B (no andaluces). Indicamos también los totales $(\mathbf{T})$. 
- En general, ¿está presente Andalucía en sus clases? ${ }^{17}$

Sí, con temas

específicos

Sí, con actividades

específicas

$\mathrm{P}=27 \mathrm{~S}=7$

$A=27 \quad B=7$

$\mathrm{T}=34$

Sí, como parte de

$$
\mathrm{P}=12 \mathrm{~S}=3 \quad \mathrm{~A}=13 \mathrm{~B}=2 \quad \mathrm{~T}=15
$$

otros temas o

actividades

$\mathrm{P}=28 \quad \mathrm{~S}=8$

$\mathrm{A}=31 \quad \mathrm{~B}=5$

$\mathrm{T}=36$

Regular

$\mathrm{P}=9 \quad \mathrm{~S}=1$

$\mathrm{A}=10 \quad \mathrm{~B}=0$

$\mathrm{T}=10$

Poco presente

$\mathrm{P}=15 \quad \mathrm{~S}=6$

$\mathrm{A}=19 \quad \mathrm{~B}=2$

$\mathrm{T}=21$

Nada presente

$\mathrm{P}=5 \quad \mathrm{~S}=4$

$\mathrm{A}=8 \quad \mathrm{~B}=1$

$\mathrm{T}=9$

N/C

$\mathrm{P}=1 \quad \mathrm{~S}=0$

$\mathrm{A}=1 \quad \mathrm{~B}=0$

$\mathrm{T}=1$

- ¿Hay en su clase (en caso de tener varias, de aquella de la que sea tutor/a) algún símbolo ideutificativo de...? (Poner una $X$ en cada caso afirmativo) ${ }^{18}$.

La localidad
España
Europa
Andalucía
N/C

$$
\begin{array}{ll}
P=23 & S=2 \\
P=24 & S=6 \\
P=10 & S=6 \\
P=34 & S=5 \\
P=46 & S=15
\end{array}
$$$$
\mathrm{A}=22 \quad \mathrm{~B}=3
$$$$
\mathrm{T}=25
$$$$
\mathrm{A}=23 \mathrm{~B}=7
$$$$
\mathrm{T}=30
$$$$
\mathrm{A}=13 \mathrm{~B}=3
$$$$
\mathrm{T}=16
$$$$
\mathrm{A}=34 \mathrm{~B}=5
$$$$
\mathrm{T}=39
$$$$
\mathrm{A}=57 \mathrm{~B}=4
$$$$
\mathrm{T}=61
$$

- ¿Considera necesaria la presencia de tales símbolos en clase? (Poner una $X$ en cada caso afirmativo $)^{19}$.

$\begin{array}{lllll}\text { De la localidad } & \mathrm{P}=45 \mathrm{~S}=6 & \mathrm{~A}=46 \mathrm{~B}=5 & \mathrm{~T}=51 \\ \text { De España } & \mathrm{P}=39 \mathrm{~S}=7 & \mathrm{~A}=39 \mathrm{~B}=7 & \mathrm{~T}=46 \\ \text { De Europa } & \mathrm{P}=27 \mathrm{~S}=12 & \mathrm{~A}=35 \mathrm{~B}=4 & \mathrm{~T}=39 \\ \text { De Andalucía } & \mathrm{P}=32 \mathrm{~S}=14 & \mathrm{~A}=60 \mathrm{~B}=6 & \mathrm{~T}=66 \\ \text { Ninguno } & \mathrm{P}=39 \mathrm{~S}=10 & \mathrm{~A}=41 \mathrm{~B}=8 & \mathrm{~T}=49 \\ \text { N/C } & \mathrm{P}=1 \quad \mathrm{~S}=1 & \mathrm{~A}=2 \quad \mathrm{~B}=0 & \mathrm{~T}=2\end{array}$

17. A veces, las respuestas a esta pregunta han sido más de una. En estos casos, hemos considerado la que más se acerca a la consideración de mayor presencia de Andalucía en sus clases.

18. La suma de respuestas supera el número de encuestados (126), puesto que pueden responderse a más de nn ítem. Ofrecemos el total para cada ítem, ya que puede ser ilustrativa la proporción de cada uno respecto al total de encuestados.

19. Ver nota 18.

20. Ver nota 18. 
- ¿A quién acude preferentemente para la organización de actividades culturales? ${ }^{20}$

\begin{tabular}{|c|c|c|c|}
\hline Ayuntamiento & $\mathrm{P}=37 \mathrm{~S}=7$ & $\mathrm{~A}=38 \mathrm{~B}=6$ & $\mathrm{~T}=44$ \\
\hline Junta & $P=20 \quad S=7$ & $A=24 \quad B=3$ & $\mathrm{~T}=27$ \\
\hline Otros (especificar) & $P=42 \quad S=11$ & $\mathrm{~A}=44 \mathrm{~B}=9$ & $\mathrm{~T}=53$ \\
\hline $\mathrm{N} / \mathrm{C}$ & $\mathrm{P}=23 \quad \mathrm{~S}=6$ & $\mathrm{~A}=25 \mathrm{~B}=4$ & $\mathrm{~T}=29$ \\
\hline
\end{tabular}

- ¿Dedican suficiente espacio los libros de texto a Andalucía?

$\begin{array}{llll}\text { Suficiente } & \mathrm{P}=44 \mathrm{~S}=10 & \mathrm{~A}=42 \quad \mathrm{~B}=12 & \mathrm{~T}=54 \\ \text { Insuficiente } & \mathrm{P}=39 \mathrm{~S}=12 & \mathrm{~A}=48 \quad \mathrm{~B}=3 & \mathrm{~T}=51 \\ \text { Demasiado } & \mathrm{P}=0 \quad \mathrm{~S}=2 & \mathrm{~A}=2 \quad \mathrm{~B}=0 & \mathrm{~T}=2 \\ \text { N/C } & \mathrm{P}=14 \mathrm{~S}=5 & \mathrm{~A}=17 \quad \mathrm{~B}=2 & \mathrm{~T}=19\end{array}$

- ¿Considera que la materia que imparte debe ser enfocada de la misma manera en Andalucía que, por ejemplo, en Madrid? 